\title{
Recours inappropriés aux urgences pédiatriques par les parents : approche qualitative d'un comportement multidimensionnel
}

\section{Inappropriate use of Paediatric Emergency Services by the parents: a qualitative approach of a multidimensional behaviour}

\author{
Reçu le 6 septembre 2017; accepté le 6 février 2018 \\ (C) SFMU et Lavoisier SAS 2018
}

J.-P. Canévet · C. Bourgogne $\cdot$ B. Querbès $\cdot$ M. Jourdain

\author{
B. Vrignaud · C. Gras-Leguen
}

Résumé Introduction : Les recours aux services d'urgences pédiatriques se multiplient, mais une partie d'entre eux sont médicalement inappropriés. Pour comprendre, au-delà des déterminants connus comme la démographie médicale ou l'attraction du plateau technique, la genèse de ces recours inadéquats et les logiques internes des parents concernés, une enquête a été menée en Pays de la Loire.

Méthode : Qualitative par entretiens semi-directifs, à Nantes et à La Roche-sur-Yon, auprès de parents ayant conduit leur enfant aux urgences et qui, après examen clinique, n'a eu besoin ni de soin ni d'investigation technique. Les entretiens étaient centrés sur le récit de l'épisode ayant motivé la décision de recours et sur le contexte familial de cette décision. Une analyse thématique puis conceptuelle a tenté d'aboutir à une typologie de recours.

Résultats : Les thèmes récurrents permettant d'interpréter ces recours concernaient : 1) le niveau d'inquiétude lié au caractère inhabituel ou inexplicable des premiers symptômes ; 2) la grande confiance accordée aux services d'urgences, effaçant tous les inconvénients possibles ; 3) une perception contrastée ou une méconnaissance du réseau de soins primaires freinant son usage non programmé. Des thèmes discordants concernant le contexte familial ont permis d'isoler trois modalités de recours inappropriés : recours par effacement parental et délégation de responsabilité de la part de parents se position-

J.-P. Canévet $(\bowtie) \cdot$ C. Bourgogne · B. Querbès · M. Jourdain ·

C. Gras-Leguen

Département de Médecine générale, Université de Nantes,

1, rue Gaston-Veil, F-44000 Nantes, France

e-mail : jean-paul.canevet@univ-nantes.fr

B. Vrignaud

Service de pédiatrie, CHU de Nantes,

7, quai Moncousu, F-44093 Nantes cedex 01, France

C. Gras-Leguen

Service de pédiatrie, CHU de Nantes,

38 boulevard Jean-Monnet, F-44093 Nantes cedex 01, France nant comme profanes incompétents, recours par confrontation de compétence avec revendication de reconnaissance de l'expertise parentale, recours par recherche de complémentarité de compétence avec les médecins.

Conclusion : Certains recours inappropriés peuvent cacher une demande implicite d'étayage urgent de la parentalité.

Mots clés Urgences pédiatriques · Médecine générale · Recours aux soins · Comportement en matière de santé · Études qualitatives

Abstract Introduction: Parents increasingly visit paediatric emergency services, although some visits are medically inappropriate. In order to understand the origin of these inappropriate visits and the internal logics of parents, beyond previously identified determinants such as medical demography or attraction towards technical platform, a qualitative study was conducted in the Pays de la Loire area.

Procedure: Qualitative study with semi-directive interviews was conducted in Nantes and La Roche-sur-Yon. Participants were parents who had brought their child to the emergency departments, who were not in need of any particular care or technical investigation after clinical examination. The interviews focused on the narration of the episode that motivated the decision to visit the emergencies, and on the family context of the decision. Thematic and conceptual analyses attempted to define a typology of visit.

Results: The main recurrent themes allowing interpretations of inappropriate visit were: the level of anxiety related to the unusual or unexplainable nature of the preliminary symptoms; the trust in the emergency services, disregarding any possible inconveniences; a contrasted perception or misconception of the primary care network, hence delaying its unplanned use. Conflicting themes regarding the family context have helped to isolate three modalities of inappropriate visit: parental abdication and delegation of responsibility as parents considered themselves profane or incompetent; 
confrontation of competence associated with a claim of parental expertise acknowledgement; search for complementarity of competence with the physicians.

Conclusion: Certain inappropriate visits to paediatric emergencies may mask an implicit demand of urgent support to parenthood.

Keywords Paediatric emergencies - General practice · Care-seeking · Health behaviour · Qualitative studies

L'augmentation des recours aux services d'urgences pédiatriques de l'ordre de $5 \%$ par an préoccupe les praticiens qui y travaillent [1-3]. Une part de ces recours, surtout s'ils sont autoréférés, ne se justifie pas du point de vue médical s'ils ne nécessitent ni plateau technique ni hospitalisation. Ainsi, en 2013, sur 2201 enfants de moins de 18 mois admis aux urgences sur une journée en France, $24 \%$ auraient pu, sans perte de chance, être vus en médecine générale dans la journée et $21 \%$ le lendemain [4].

Les études explorant les causes de ce phénomène mettent en avant la visibilité et la disponibilité des urgences hospitalières ainsi que les difficultés d'accès à l'offre de soins de premier recours [5].

Mais ces déterminants objectifs se doublent de facteurs subjectifs qui confèrent à la notion d'urgence une dimension profane pourvoyeuse de recours dits inappropriés, résultant d'un processus complexe où interviennent les caractéristiques du parcours de soins d'amont et le contexte familial, psychologique et social de ces recours [6]. Pour approcher ce comportement de santé, une enquête qualitative, inspirée de la sociologie compréhensive, a été menée. Son objectif était de recueillir le point de vue des parents pour explorer la genèse du sentiment d'urgence, les logiques internes et le sens qu'ils donnent à leur recours dit inadéquat. Ce type d'enquête exploratoire, qui ne teste pas d'hypothèse explicative, tente de comprendre les processus à l'œuvre pour produire le phénomène étudié. Cette démarche, productrice d'hypothèses en amont des enquêtes quantitatives causalistes, rejoint celle de la médecine narrative initiée par Charon, dont le but est de mieux comprendre le sens de l'expérience des patients $[7,8]$.

\section{Méthode}

Les parents ayant conduit spontanément et par leurs propres moyens leur enfant de moins de 15 ans et 3 mois dans un service d'urgence pédiatrique et qui n'a fait l'objet, après examen clinique, ni de soins sur place, ni de recours au plateau technique, ni d'hospitalisation.

Des parents, recrutés de février à octobre 2016, au CHU de Nantes et au Centre Hospitalier de La Roche-sur-Yon, par les pédiatres urgentistes qui ont jugé qu'une prise en charge ambulatoire aurait été suffisante. La méthode qualitative destinée à comprendre des logiques internes des acteurs n'exige pas la représentativité de l'échantillon mais sa diversité, permettant de recueillir le plus possible de modalités du phénomène étudié. Les informations issues des entretiens sont validées non par le nombre d'occurrences mais par leur contexte. Cette diversité reposait sur l'âge des parents et de l'enfant, la taille de la fratrie, les niveaux d'étude et socioéconomiques, le motif de recours, la zone d'habitat. La taille de l'échantillon n'était pas déterminée à l'avance : le recrutement pour une enquête qualitative s'arrête au point de « saturation » lorsque les informations obtenues lors des derniers entretiens sont redondantes [9]. Il a été remis, sur place, aux parents sollicités un courrier d'informations les invitant à une entrevue de recherche, sur le thème des urgences pédiatriques, et sollicitant leur consentement. Le protocole d'enquête a été validé par le Groupe nantais d'éthique dans le domaine de la santé du CHU de Nantes.

Deux investigatrices (une par site) en thèse d'exercice ont recueilli des entretiens semi-dirigés centrés sur le récit de l'épisode ayant motivé le recours aux urgences le jour du recrutement. La grille d'entretien a été élaborée en commun par les investigatrices, un enseignant senior de médecine générale et une pédiatre urgentiste, puis testée lors de deux entretiens d'essai non inclus dans les résultats. Elle invitait à « raconter » ce qui s'était passé depuis l'apparition des symptômes de l'enfant. Elle comprenait aussi des questions permettant d'explorer les comportements de santé des parents, leurs modes habituels de recours aux soins, le contexte familial et les événements de santé antérieurs éventuels vécus par eux ou leurs proches. Les entretiens ont été intégralement enregistrés et retranscrits, puis ont fait l'objet d'une analyse thématique par codage manuel et triangulé des deux investigatrices et d'un enseignant senior de médecine générale, puis d'une analyse interprétative par « catégories conceptualisantes ", pour aboutir à une typologie des recours, selon les règles de l'analyse qualitative $[10,11]$.

\section{Résultats}

L'échantillon a réuni les parents de 17 enfants, huit Nantais et neuf Yonnais, respectivement désignés par la lettre $\mathrm{N}$ ou $\mathrm{R}$ suivie du numéro d'ordre de l'entretien (Tableau 1). L'interlocuteur unique ou principal était la mère pour 14 entretiens. Les entretiens se sont tous tenus dans le mois suivant le recrutement et, selon leur souhait, au domicile des parents. Ils ont duré entre 30 minutes et une heure. Les investigatrices ont fait face à une dizaine de refus pour mauvaise compréhension, indisponibilité ou raisons personnelles.

La perception angoissante des symptômes était largement partagée : l'expérience des parents était dominée par la peur, mot prononcé dix fois dans l'entretien R8 et 11 fois par Mme 


\begin{tabular}{|c|c|c|c|c|c|c|c|c|c|c|c|c|c|}
\hline$\cong$ & 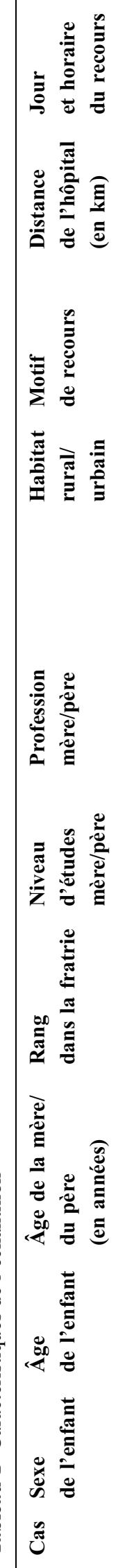 & 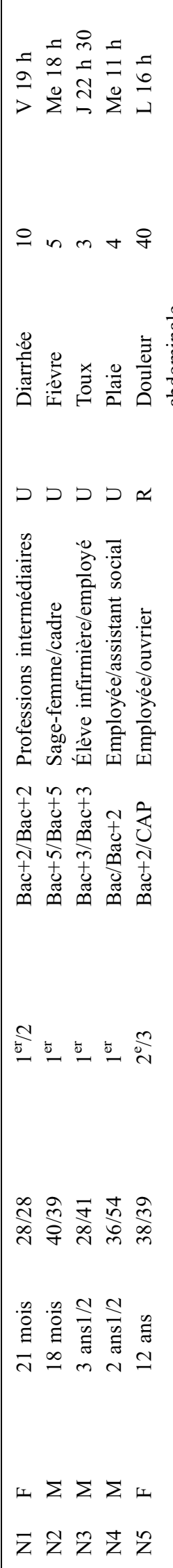 & 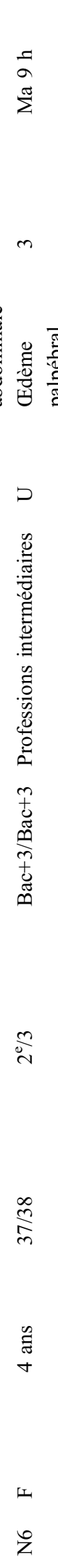 & 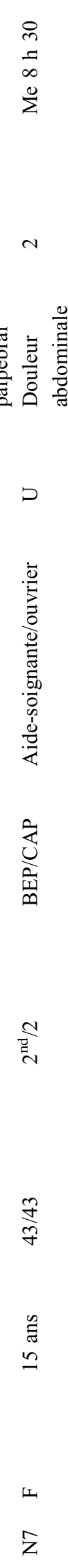 & 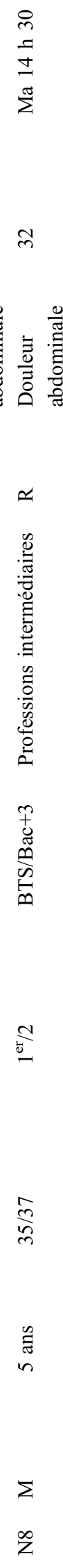 & 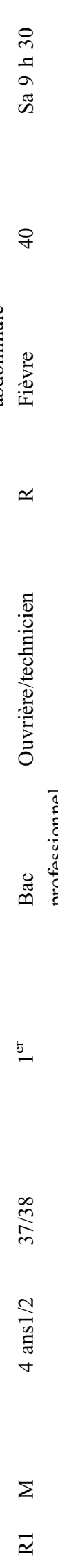 & 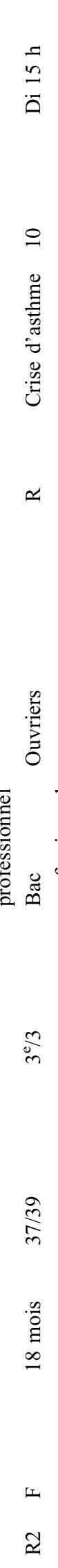 & 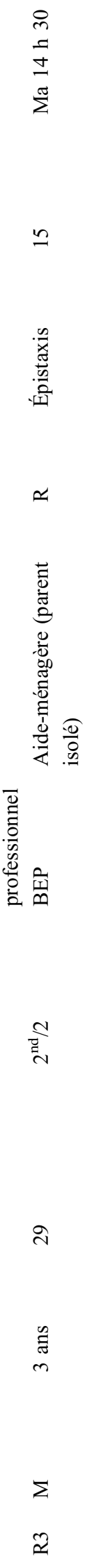 & 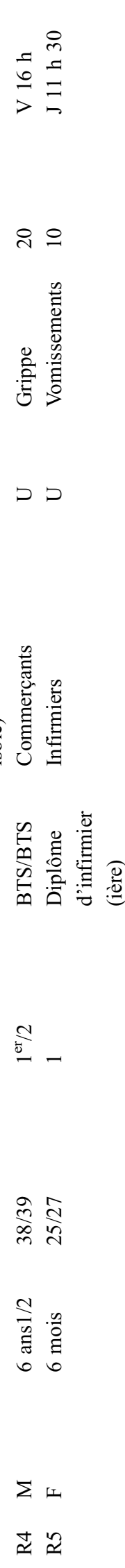 & 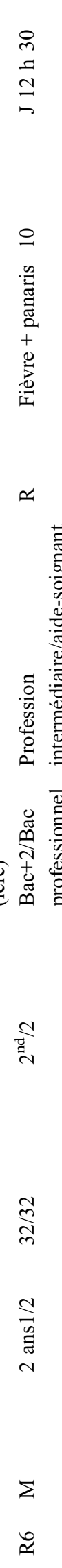 & 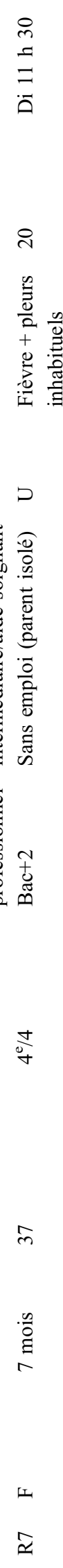 & 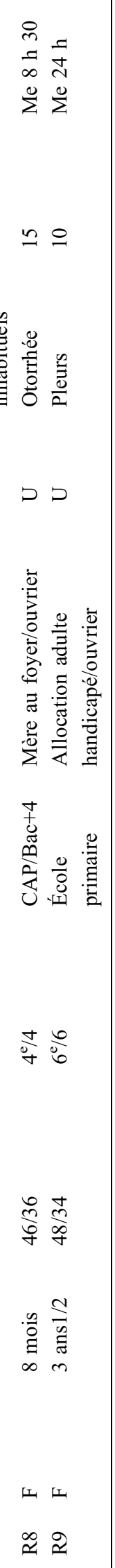 & 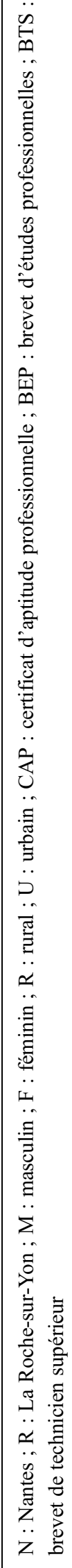 \\
\hline
\end{tabular}


R3, et le stress mentionné 15 fois par Mme N2. Cette perception était liée soit à la douleur de leur enfant, insupportable pour les parents, et autant redoutée que la gravité éventuelle du symptôme (N7), soit à l'organe ou la fonction en cause : «Un saignement de nez pour moi c'est pas anodin, ça touche la tête » (R3), «L'œil... ça a un côté hyperfragile, et on s'inquiète déjà un peu plus » (N6), «Elle mangeait pas, elle voulait pas boire, ... surtout que ça reste des choses importantes à la vie » (N1). L'alarme pouvait aussi provenir du caractère inhabituel ou dépourvu de sens d'un symptôme : «Voir son enfant, euh..., ne plus être le même et puis ne pas comprendre ce qui se passe » (N1), ou N5 déconcerté par une gastroentérite banale de sa fille, habituellement constipée : "C'est rare ... c'est plutôt l'inverse, alors je me suis dit il y a un truc qui dec ... dans le bide » et «alors qu'elle est pas douillette, elle se plaignait beaucoup », ajoutant: « Je ne savais pas qu'on faisait des gastro en été, alors j'ai jamais pensé à ça ». Mme R8, à propos de l'otorrhée «jaune» de sa fille, est revenue à trois reprises sur le fait qu'elle " $n$ 'avait jamais vu ça dans sa vie, en fait, ça me fait peur, en fait ». En l'absence de sensation d'urgence, la durée du trouble a pu conduire à un sentiment d'inaction coupable imposant soudain une action immédiate : "ça faisait quelques jours que ça durait, moi je me suis dit : je passe peut-être à côté de quelque chose ... j'allais pas non plus rester indéfiniment comme ça avec une petite qui mangeait plus » (N7). Quels que soient les symptômes, l'anticipation anxieuse d'une aggravation colorait la plupart des entretiens. Le lexique des catastrophes était alors utilisé : « exploser », « éclater », « terrible» « mort»: «Je vois le pire, une méningite, une septicémie ... il y a un gros stress, c'est l'angoisse de mort ... dès qu'on se stresse y a cette angoisse qui plane " (N2). "L'otite peut exploser, percer le tympan, après elle va perdre l'audition, quoi! Après toute la nuit j'ai pas dormi» (R8). Et Mme N6 se défiant d'elle-même voulait écarter le risque de : "passer à côté d'un truc ... plus grave que j'imaginais être. Je me suis dit c'est peut-être l'appendicite après, euh, si c'est pas pris à temps ça peut éclater je crois à l'intérieur $\gg(\mathrm{N} 8)$.

Le souvenir d'une expérience antérieure traumatisante exacerbant l'inquiétude a été rapporté à plusieurs reprises : « J'ai vu ma cousine, ... j'étais petite à cette époque-là ... c'est compliqué parce qu'elle a perdu la vie dans mes bras » (R7). "J'ai eu un peu peur parce que ma maman a fait un panaris l'année dernière et ça a fini au bloc ... et très mal... Donc, tout de suite j'ai pensé à ça », disait Mme N6 à propos de l'œè̀me palpébral de sa fille de quatre ans, qu'elle avait déjà vue « vraiment en péril » à l'âge de trois semaines pour une fièvre. Mme N7 se reprochant un précédent retard diagnostique d'ostéomyélite pour son aînée, et forte du souvenir de sa propre appendicite, a conduit sa fille aux urgences, car « si jamais elle fait une appendicite que je passe à côté, qu'elle finit par une péritonite, alors là non, non ».
Le sentiment d'impuissance devant le jeune enfant qui ne peut pas verbaliser ses symptômes majorait cette mise à l'épreuve de la responsabilité parentale : «Les enfants, on sait jamais sur quel pied danser. C'est compliqué, très compliqué», "presque 40, pour nous, pour moi, on sait ce qu'il faut faire, mais lui c'est pas pareil » (R4). Mme R2, asthmatique comme sa fille, « gère » pour elle-même, mais pour sa fille « c'est impressionnant. Surtout ma petite vient d'avoir 18 mois ».

Les services d'urgences étaient au moins autant sollicités pour une prestation technique que comme point d'appui pour étayer le sentiment de compétence parentale. Tous les entretiens ont révélé un besoin prioritaire d'apaisement de l'inquiétude, autant que de soin. L'expression «besoin d'être rassuré(e) » était récurrente : "De toute manière, de moimême, je serais quand même allée aux urgences, histoire d'être rassurée et de savoir » (Mme R3, parent isolée). La décision de recours n'était pas freinée par l'intuition de bénignité des symptômes. À celle-ci devait se substituer une certitude validée par l'autorité médicale : «J'ai une part de rationalité qui me dit que c'est pas si grave que ça, mais faut qu'on me rassure "( $(\mathrm{N} 2)$. Après l'apaisement, les parents cherchaient à " comprendre » le sens du symptôme (N1) ou d'une situation qui leur échappait, grâce à « un diagnostic plus précis aux urgences » (N7).

Le recours au plateau technique était souvent évoqué, non comme une nécessité dans la situation présente, mais comme une éventualité, "si besoin ", "si ça avait été plus grave ». Et en contradiction avec le motif médicalement inadapté du recours, le service d'urgences était parfois bien perçu comme le lieu adéquat "lorsque c'est vital ou qu'il y a quelque chose de cassé » (R4). Pour les parents rencontrés, les services d'urgences représentaient l'idéal de la compétence attendue pour susciter la confiance : «Ils sont à la pointe » (N2). «Ils sont habitués, c'est le CHU d'une grande ville » (N3). «C'est là où il y a les personnes les plus compétentes » (N5), «les plus passionnées » aussi (R5) qui ont «vraiment bien ausculté de partout, du coup ça m'a rassuré, là c'est sûr qu'il y avait rien » (N4). La visibilité et l'accessibilité étaient aussi plébiscitées, en opposition à l'indisponibilité de l'offre de soins ambulatoires. L'absence d'avance d'argent a été évoquée deux fois. La brièveté de la consultation ou le défaut d'explications ou le sentiment d'être « une personne dans la masse » (N3) ou le risque de contagion en salle d'attente ont été parfois évoqués, mais la qualité de l'accueil, l'écoute accordée ou les consignes orales ou écrites reçues ont été largement appréciées.

Devant tant de qualités attribuées aux urgences hospitalières, les contrepoids susceptibles de limiter les recours étaient peu opérants. Ainsi, l'attente était supportable : "Même si on doit attendre une demi-journée, ça ne me choque pas » (R4), «ça fait partie du jeu » (N5). Cette attente apparaissait même comme un indice du caractère 
impropre du recours : «Si on attend, c'est bon signe, finalement, c'est que c'est pas très grave » (R6). L'expérience familière du réseau médical habituel n'était pas non plus un frein au recours hospitalier, car les patients en avaient des perceptions contrastées : les qualités humaines de leur généraliste étaient appréciées ainsi que son expérience et sa bonne connaissance du milieu familial. Mais les délais de rendez-vous, l'absence de consultations non programmées, le barrage par les secrétaires, le manque de disponibilité pour des conseils téléphoniques, les retards en consultation, la difficulté à trouver un médecin traitant pour ceux qui n'en avaient pas, la brièveté des consultations et les désaccords entre médecins ont été abondamment critiqués. Ces déceptions ont conduit les parents, le plus souvent, à ne pas chercher de solution ambulatoire, même dans la journée. Mais, malgré un rendez-vous proposé le jour même, Mme R5, mère d'un ancien prématuré, n'a pas pu supporter ce délai, ni Mme R7, en situation de précarité, hébergée en foyer et en proie à un sentiment permanent d'insécurité. Parfois, un premier recours ambulatoire adéquat mais non suivi d'amélioration a semé le doute : «Je vais pas retourner voir mon médecin traitant une troisième fois, je vais passer pour une cinglée » (N1). La notion de parcours de soins, mal comprise, pouvait être un facteur de confusion : "On sait pas qui appeler » (N6). Et l'organisation des soins non programmés était mal connue : pour Mme N4, "Le tout début c'est le pharmacien ... puis ensuite le médecin généraliste si c'est possible, puis après y a le flou entre le 15, les pompiers, SOS médecins ... je vois pas trop la différence en degré d'urgence. Et puis le CHU où on se déplace... ». Les maisons médicales de garde sont le plus souvent ignorées ou négligées, car «le médecin de garde, qu'est-ce qu'il va nous dire? D'aller aux urgences s'il y a vraiment un souci»(R1). Le recours à la régulation médicale du 15 , bien connu, était souvent considéré comme un ultime recours en cas d'urgence grave.

D'autres thèmes, moins partagés, ont permis d'identifier trois modalités différentes de recours inappropriés. Des conceptions diversifiées de la fonction parentale en constituent l'axe principal. Ces modalités ne sont pas exclusives les unes des autres, mais la prévalence ou l'intensité de certains thèmes au sein des récits ont permis de les distinguer.

Des recours par effacement parental : plusieurs parents ont exprimé une difficulté à supporter le poids de la responsabilité parentale devant les symptômes inhabituels et inquiétants de leur enfant (R1, R7, N2, N3). En se rendant aux urgences, ils souhaitaient déléguer au plus vite leur responsabilité aux soignants en se positionnant comme profanes incompétents. R1, ouvrière qui « a suivi les ordres $d u$ médecin », insistait sur son "impuissance » devant son enfant malade. Malgré ses initiatives (consultation médicale et d'une guérisseuse), elle doutait de ses capacités et s'en remettait en toute confiance aux urgentistes. R7, fragilisée par un passé de violences conjugales, exprimait un sentiment d'incompétence et un besoin immédiat de voir un médecin ; « Je sais que c'est pas grave, mais j'en ai besoin ». Les parents $\mathrm{N} 2$ - lui survivant d'une très grave maladie, elle tombée enceinte difficilement - se sentaient très inexpérimentés, doutant de leur capacité à protéger cet enfant inespéré. Ils s'en remettaient immédiatement aux experts qui avaient déjà sauvé M. N2 : "Pour moi et pour mon mari qui a eu tous ces malheurs, voilà, c'est CHU... ». Mme N3, élève infirmière, doutant de sa compétence aussi bien comme soignante que comme mère, parlait, en hésitant, du soulagement éprouvé à s'en remettre aux professionnels pour prendre soin de son enfant.

Des recours par confrontation d'expertise : d'autres parents se sont affirmés comme l'expert de leur enfant, attendant des professionnels une reconnaissance de leur compétence. Ils ont élaboré un diagnostic profane et une conviction de gravité du cas. Leur recours ne souffrait aucun doute. Mme N1, sentant sa compétence maternelle dépréciée par un médecin de ville, s'est rendue aux urgences pour comprendre les symptômes de sa fille et pour être prise en considération plus que pour obtenir des soins. M. N5, sensibilisé par les troubles fonctionnels digestifs anciens de sa fille, convaincu qu'elle souffrait d'appendicite aiguë, a pensé immédiatement aux complications les plus sévères. Sa conviction anxieuse ne souffrait aucun retard pour la prise en charge. Pour M. et Mme R2, familiers de la maladie asthmatique et convaincus de la nécessité de lui administrer des aérosols, l'hospitalisation de leur fille s'imposait comme une évidence. L'inquiétude de Mme N7, appuyée sur son expérience d'aide-soignante et sur le souvenir d'un retard diagnostique d'ostéomyélite pour son aînée, a dicté son recours sans hésitation.

Des recours par complémentarité de compétence : ces parents s'inscrivaient dans un mode de comportement conforme à la logique médicale si ce n'était la bénignité du cas de leur enfant. Ils ont cherché une expertise professionnelle complémentaire à la leur : ils se sentaient capables de faire face aux premiers symptômes et s'en remettaient très vite aux médecins, animés par une foi en l'efficacité de la médecine en toute circonstance. Ils ont parfois tenté vainement de contacter leur médecin traitant pour une consultation immédiate, et par souci de sécurité, ils se sont tournés vers les urgences hospitalières qu'ils estimaient le plus immédiatement efficaces (N4, N6, N8, R3, R4, R5, R6, $\mathrm{R} 8, \mathrm{R} 9)$.

\section{Discussion}

Le résultat principal de cette étude a trait au caractère multidimensionnel des recours dits médicalement inappropriés aux services d'urgences pédiatriques. Les déterminants déjà 
connus, telles l'indisponibilité des praticiens du premier recours ou l'image positive de l'hôpital, sont retrouvés $[2-5,7]$.

Des éléments d'explication supplémentaires sont apparus, en particulier une frustration, face au médecin traitant qui ne répond pas à toutes les attentes, que deux enquêtes qualitatives, australienne et américaine, avaient déjà mise en évidence $[12,13]$. L'évolution sociologique décrite par Peneff vers la recherche privilégiée d'une réponse médicale la plus armée et rapide possible y est retrouvée ainsi que l'affaissement du sentiment de compétence parentale que Dubos et Codaccioni ont attribué à la médicalisation de la puériculture $[14,15]$. Mais ces déterminants de type culturels ou sociologiques se conjuguent à des facteurs subjectifs qui donnent un sens singulier à ces recours, y compris dans leurs aspects les plus surprenants, comme pour Mme N1 venue pour « être écoutée ». Le sentiment d'impuissance face à des symptômes inhabituels, l'inexpérience parentale ou les expériences traumatisantes antérieures participent à la genèse de l'anxiété et du sentiment d'urgence qui donnent une justification aux recours dits inappropriés du seul point de vue des soins techniques. Insatisfaisants pour des soignants surchargés, les recours inadéquats peuvent aussi être lus comme un mode d'expression du rôle parental face aux soignants et être parfois entendus comme une demande muette d'aide face à un sentiment d'incompétence parentale qui pourrait bénéficier d'une réorientation vers les professionnels adéquats.

La discordance apparue entre attente des parents et missions spécifiques des urgentistes témoigne du « malentendu entre ce qui fait le travail du médecin et ce qui fait l'objet de la préoccupation des parents » que Hardy a décrit de façon superposable dans le champ de l'oncologie pédiatrique et qui constitue un défi permanent pour les soignants [16].

La validité des résultats de cette étude exploratoire qualitative est étayée par l'homogénéité des résultats obtenus sur les deux sites d'enquête, par la méthode d'analyse des données triangulée entre les investigateurs et par la cohérence des résultats avec les données de la littérature. Mais le recrutement indirect des interviewés, par l'entremise des urgentistes impliqués dans leur accueil, a pu constituer une limite : dans ces situations, les cas les plus banaux ont pu passer plus inaperçus et être moins sollicités.

\section{Conclusion}

Complexe et multidimensionnel, le sentiment d'urgence, nourri de l'histoire singulière des patients, contribue à l'encombrement des services d'urgence pédiatrique : une demande implicite d'étayage de la parentalité alimente une partie des recours dits inappropriés aux urgences pédiatriques. Ce constat, complétant les enquêtes par questionnaire, encourage à interroger l'organisation des soins ambulatoires non programmés, adéquats pour traiter ces recours ne nécessitant pas d'intervention technique, ainsi qu'à expérimenter et évaluer, en amont de la demande de soins, des mesures d'éducation en santé susceptibles de consolider le sentiment de compétence parentale.

Liens d'intérêts : les auteurs déclarent ne pas avoir de lien d'intérêt.

\section{Références}

1. Gras-Leguen C, Vrignaud B, Levieux K (2015) Organisation des soins aux urgences pédiatriques. Rev Prat 65:627-8

2. Leclerc F, Martinot A, Sadik A (1999) L'inflation des urgences pédiatriques. Arch Pediatr 6:454-6

3. Vuagnat A (2013) Les urgences hospitalières qu'en sait-on ? In: DREES (ed) Le panorama des établissements de santé 2013. DREES Éditions, Paris, pp 9-28

4. Ministère de la Santé (2015) Enquête nationale sur les structures d'urgence hospitalière. Tableau 6. L'appréciation de l'adéquation du recours aux urgences selon la tranche d'âge du patient. http:// www.data.drees.sante.gouv.fr/TableViewer/tableView.aspx? Reportld=1201 (Dernier accès le 17 août 2017)

5. Boisguérin B, Valdelièvre H (2014) Urgences : la moitié des patients restent moins de deux heures, hormis ceux maintenus en observation. DREES Études et Résultats 889:1-7

6. Gentile S, Amadeï E, Bouvenot J (2004) Attitudes et comportement des usagers face à une urgence réelle ou ressentie. Santé publique 16:63-74

7. Cour des Comptes (2014) Les urgences hospitalières : une fréquentation croissante, une articulation avec la médecine ambulatoire à repenser. In: Rapport sur l'application des lois de financement de Sécurité sociale. Cour des comptes Ed., Paris, pp 350-77

8. Charon R (2001) Narrative medicine. A model for empathy, reflection, profession, and trust. JAMA 286:1897-902

9. Blanchet A, Gotman A (2003) L'enquête et ses méthodes : l'entretien. Nathan Université Ed., Paris, 127 p

10. Savoie-Zajc L (2004) Technique de validation par triangulation. In: Mucchielli A (ed) Dictionnaire des méthodes qualitatives en sciences humaines. A Colin $2^{\mathrm{e}}$ ed, Paris, pp 289-90

11. Paillé P, Muchielli A (2012) L'analyse qualitative en sciences humaines et sociales. A Colin $3^{\mathrm{e}}$ Ed., Paris, pp 315-73

12. Woolfenden S, Ritchie J, Hanson R, et al (2000) Parental use of a paediatric emergency department as an ambulatory care service. Aust NZ J Public Health 24:204-6

13. Berry A, Brousseau D, Brotanek JM, et al (2008) Why do parents bring children to the emergency department for non-urgent conditions? A qualitative study. Ambul Pediatr 8:360-7

14. Peneff $\mathrm{J}$ (2000) Les malades des urgences, une forme de consommation médicale. Métallié Ed., Paris, $189 \mathrm{p}$

15. Dubos JP, Codaccioni X (2000) L'éducation pour la santé en maternité. Arch Pediatr 7:299-301

16. Hardy AC (2013) Travailler à guérir. Sociologie de l'objet du travail médical. Presses de l'EHESP Ed., Rennes, pp 197-221 